\title{
In vitro and in vivo Permeation of Vitamin E and Vitamin E Acetate from Cosmetic Formulations
}

\author{
Aly Nada ${ }^{a} \quad$ Yellela S.R. Krishnaiah ${ }^{\mathrm{a}, \mathrm{b}} \quad$ Abdel-Azim Zaghloul ${ }^{\mathrm{a}} \quad$ Ibrahim Khattab $^{\mathrm{a}}$ \\ a Department of Pharmaceutics, Faculty of Pharmacy, Kuwait University, Jabriya, Kuwait; \\ bUS Food and Drug Administration, Silver Spring, Md., USA
}

\section{Key Words}

Vitamin E • Vitamin E acetate $\cdot$ Cosmetics $\cdot$ Permeation .

Neonatal rats

\begin{abstract}
Objective: To investigate the ability of $\alpha$-tocopherol acetate (TA) and $\alpha$-tocopherol (T), widely used ingredients in cosmetics, to cross the epidermal barrier using the neonatal rat as a model. Materials and Methods: The content of T and TA in four marketed products (A-D) and two experimental formulations (F1, F2) was investigated by HPLC. An in vitro permeation study was performed in neonatal rat epidermis using diffusion cells. In vivo permeation was studied in neonatal rats after repeated application of the products and analysis of T and TA in the stratum corneum/deeper skin layers. Results: Variable contents of TA were found in the marketed products $(0.12-0.53 \%)$. No vitamin permeation was detected through the stratum corneum as in vitro biological barrier after $4 \mathrm{~h}$. No detectable T and TA were seen in the in vivo permeation study in the epidermis. Variable degrees of drug penetration (4.3-12.6\%) of the applied dose into the deeper skin layers were observed, depending on the formulation. In vivo application of TA-containing preparations did not result in any transformation of TA into T under the de-
\end{abstract}

scribed experimental conditions. Conclusion: TA and T exhibited variable skin penetration and TA did not transform into $T$ under the experimental conditions. The data underscored the need for further studies to optimize such formulations to improve vitamin $\mathrm{E}$ transdermal permeation and eventually achieve the expected cosmetic/therapeutic outcome.

Copyright $\odot 2011$ S. Karger AG, Basel

\section{Introduction}

Inclusion of botanical extracts, vitamins and antimicrobials into cosmetics has become an important marketing advantage, mostly without scientific proof. Claims based on these additives must be carefully phrased to maintain the product(s) in the cosmetic area. Although these substances exhibit systemic effects upon ingestion, evidence for skin benefits from topical application has not been established [1]. Patients often seek over-thecounter products due to market availability, comparably cheaper prices, and the lack of the physician bottleneck.

Vitamin E ( $\alpha$-tocopherol, $\mathrm{T}$ ) is one of the widely used ingredients in over-the-counter products for protection against skin ageing. $\mathrm{T}$ is a lipid-soluble antioxidant which 
plays key roles in protecting cell membranes from lipid peroxidation by free radicals [2, 3]. Furthermore, Gensler and Magdaleno [4] have shown that, in terms of parameters of tumor incidence and tumor burden, T significantly reduces photocarcinogesis. $\mathrm{T}$ is normally distributed in skin, with the highest levels in the deepest layers [5]. It has been documented that $\mathrm{T}$ is the major antioxidant in the human epidermis, and that its depletion is an early and sensitive marker of environmental oxidative damage [6]. Ricciarelli et al. [7] pointed out that $\mathrm{T}$ reduced the age-dependent increase of collagenase expression by inhibiting protein kinase $\mathrm{C}$ activity. The protective effects of $\mathrm{T}$ against photoaging have been demonstrated in various animal and in vitro skin models [8-11]. Despite the media attention and consumer popularity that these ingredients have generated, there have been few scientific studies to support these claims $[12,13]$. Furthermore, a recent study on the metabolic conversion of $\alpha$-tocopherol acetate (TA) into T in skin demonstrated that permeation and metabolism of TA was highly dependent on the delivery system [14]. Although cosmetics and cosmeceuticals are tested for safety, testing to determine whether or not beneficial ingredients actually reflect a manufacturer's claims is not mandatory [15]. In addition, the stratum corneum represents the major barrier against drug delivery and is considered the limiting factor to permeation of drugs across the skin [8].

Therefore, academia and state agencies should contribute in reviewing and verifying the claims made by manufacturers to protect the consumers and ensure valid and scientifically founded claims for cosmetic products. Thus, the objectives of the present study were to assess the transdermal permeation of TA and T from the marketed and experimental products. This investigation involved four commercially available cosmetic products on the Kuwaiti market (A, B, C, D), as well as two experimental cosmetic formulations (F1, F2). Rat epidermis was used as a skin model for in vitro experiments and the neonatal rat for the in vivo study. Furthermore, the liberation of the active form of the free vitamin $(\mathrm{T})$ from the ester form following in vivo application of the above-mentioned formulations to neonatal rats was evaluated.

\section{Materials and Methods}

Materials

Methanol, acetonitrile, hexane and ethanol used in the study were of HPLC grade (Merck, Darmstadt, Germany). TA was obtained from BASF, Ludwigshafen, Germany. T, soybean oil and corn oil were procured from Sigma Aldrich Chemie $\mathrm{GmbH}$,
Table 1. Composition of experimental formulations (F1 and F2) per $100 \mathrm{~g}$ cream

\begin{tabular}{lll}
\hline Ingredients & F1 & F2 \\
\hline T, \% & 0.5 & - \\
TA, \% & - & 0.5 \\
Stearic acid, g & 6 & 6 \\
White soft paraffin, g & 4 & 4 \\
Lanoline, g & 4 & 4 \\
Soybean oil, ml & 9 & 9 \\
Corn oil, ml & 9 & 9 \\
Captex SBE, g & 4 & 4 \\
Glycerol, ml & 10 & 10 \\
Sorbitol, ml & 10 & 10 \\
Acconon S-35, g & 5 & 5 \\
Potassium hydroxide, g & 0.28 & 0.28 \\
Water, ml & 39 & 39 \\
\hline
\end{tabular}

Steinheim, Germany. Propylene glycol (Generico Medical Practice, AB Almere, Holland), stearic acid, white soft paraffin, potassium hydroxide (Loba Chemie, India), lanoline, glycerol, sorbitol (Gainland Chemical Co., UK), Captex SBE and Acconon S-35 (Abitec Corp., Janesville, Wisc., USA). All other chemicals used were of analytical grade.

Four commercial products (A, B, C, D) were obtained from retail pharmacies in Kuwait and they contained only TA, without declaration of its quantity.

\section{Preparation of Vitamin E-Containing Laboratory Products}

Since the available commercial products contained only TA without quantitative declaration of content, it was decided to prepare experimental formulations containing $\mathrm{T}$ and TA. Two cream-emulsion cosmetic formulations, each containing about $0.5 \% \mathrm{w} / \mathrm{w}$ of T/TA, were prepared in the laboratory, simulating the complex composition of commercial cosmetic preparations (table 1). The cream was prepared by melting stearic acid in a porcelain dish over a water bath $\left(75-80^{\circ} \mathrm{C}\right)$, then the semisolid ingredients (lanoline, white soft paraffin and Captex SBE) were added until all the mixture was melted, and finally soybean oil and corn oil were added (oily phase). Potassium hydroxide was dissolved in water followed by Acconon S-35, then glycerin and sorbitol were added and the mixture was heated to $75-80^{\circ} \mathrm{C}$ (aqueous phase). The aqueous phase was added to the oily phase with trituration and the mixture was then removed from the water bath and mixing was continued until a homogeneous creamy liquid was obtained. Accurately weighed amounts of $\mathrm{T}$ and $\mathrm{TA}$ were added to the mixture (avoiding addition of the vitamin to the cream while it was hot to avoid possible degradation), resulting in a nominal concentration of 0.532 and $0.539 \% \mathrm{w} / \mathrm{w}$, respectively. The final cream was filled in well-closed plastic jars.

Method for Extraction of TA and T from Cosmetic Products

Triplicate 500-mg samples of each formulation were extracted with methanol, centrifuged, and the supernatant of extract was filtered through a $0.45-\mu \mathrm{m}$ cellulose filter and injected into the HPLC for estimating T and TA as previously described [16]. 
HPLC Method for the Estimation of $T$ and TA

Waters 2690 HPLC (Waters 2690 Separations Module, Milford, Mass., USA) with variable wavelength PDA detector, a disposable guard column C-18 and RP Waters Symmetry C-18 column (4.6 $\times 150 \mathrm{~mm}, 5 \mu \mathrm{m}$ particle size) was used. The column temperature was maintained at $25^{\circ} \mathrm{C}$. Samples $(50 \mu \mathrm{l})$ were injected and the flow rate of the mobile phase (3\% v/v water/methanol) was adjusted at $1.5 \mathrm{ml} / \mathrm{min}$. The eluents were monitored at 290 and $283 \mathrm{~nm}$ for $\mathrm{T}$ and TA, respectively. The peak areas for $\mathrm{T}$ and TA were recorded and analyzed using the Millennium Software Empower from Waters. The peak areas of T and TA were subjected to regression analysis against their concentrations. The details of the procedure and validation of the method were reported previously [16].

\section{In vitro Permeation Study}

The permeation studies were performed using an automated flow-through apparatus (Perme-Gear I-Line Cells, ILC14). The rat epidermal membrane was mounted onto the dynamic diffusion cell with the stratum corneum side facing the donor compartment and the dermal side facing the receptor compartment. The diffusion cell was equilibrated overnight at $32^{\circ} \mathrm{C}$ with a continuous flow of $0.05 \mathrm{M}$ phosphate buffer ( $\mathrm{pH} 7.4)$ through the receptor compartment. The effective diffusion area was $0.5 \mathrm{~cm}^{2}$. Rat epidermis (Sprague-Dawley rats, 5-10 g) was used as a skin model for this study. The care and use of the animals was in accordance with institutional guidelines. The neonate (2-3 days old) rats were euthanized using carbon dioxide asphyxiation before the experiments, and full-thickness skin was surgically removed from each rat. Rat epidermis was prepared by a heat separation technique [17]. The entire skin of neonate rats was soaked in water at $60^{\circ} \mathrm{C}$ for $1 \mathrm{~min}$, followed by careful removal of the epidermis. Rat epidermis, so prepared, was washed with water and examined for physical damage by using a magnifying lens. Rat epidermis free from physical damage was used in the in vitro permeation study. One gram of each of the commercial or experimental cosmetic formulations was filled in the donor cell. After predetermined times $(1,2,3,4 \mathrm{~h})$, samples were collected from the receptor compartment and analyzed by HPLC method. Triplicate experiments were run for each product under investigation.

\section{In vivo Permeation Study}

In this study each preparation was applied repeatedly (every $30 \mathrm{~min}$ ) over $4 \mathrm{~h}$ on the dorsal side $\left(7-8 \mathrm{~cm}^{2}\right)$ of neonatal rats. The area of skin used in the permeation study was removed and rat epidermis was prepared (as described under 'In vitro Permeation Study'), crushed and extracted with methanol and subjected to HPLC analysis for estimating the amount of T/TA retained in rat epidermis. Triplicate experiments were run for each product under investigation.

In order to further follow up the permeation, we repeated the same in vivo experiment. We separated the full-thickness neonatal rat skin, cut it into small pieces, extracted with methanol, and centrifuged, and the filtered supernatant was assayed for $\mathrm{T}$ and TA content by the above-mentioned HPLC method. The purpose of this procedure was to assess if any vitamin permeated through the stratum corneum might have been retained in the deeper skin layers. Control experiments were run on naif animals to determine naturally present vitamin. The work described in this article was carried out in accordance with The Code of Ethics of the World Medical Association (Declaration of Helsinki) for experiments involving animals.

Permeation of T/TA from Cosmetic Formulations

\section{Results}

\section{Evaluation of TA in Commercial Products}

Results of analysis of the four commercial products revealed that the initial concentration of the vitamin ester (TA) ranged from 0.12 to $0.68 \%$. The mean concentrations (SD) were 0.12 (0.001), 0.68 (0.019), 0.53 (0.084), and $0.49 \%$ (0.034) for products A, B, C and D, respectively. No free vitamin $\mathrm{E}(\mathrm{T})$ was detected in any of the tested products.

\section{In vitro Permeation Study}

Samples collected from the receiver compartment over $4 \mathrm{~h}$ did not show any detectable amount of T and TA from any of the tested preparations.

\section{In vivo Permeation Study}

The control group (3 animals) revealed the absence of any T/TA in the skin of untreated rats. Analysis of T/TA in the rat epidermis following the repeated application of the different types of products revealed absence of T/TA too. The results presented in table 2 show that T/TA permeated to variable degrees into the deeper skin layers according to the type of formulation. The results of the in vivo study (table 2 ) show that $10-12.5 \%$ of the applied TA dose permeated across the skin from the commercial products. The commercial preparation (product A) containing the least TA concentration $(0.12 \%)$ did not result in any detectable concentration in the rat skin. Regarding the experimental preparations, $4.3 \%$ of applied $\mathrm{T}$ and $4.9 \%$ of applied TA permeated from F1 and F2 formulation, respectively. No free $\mathrm{T}$ could be detected following the application of all commercial and experimental cosmetic formulations.

\section{Discussion}

Our market survey in Kuwait regarding T/TA-containing cosmetic preparations revealed that no one product carries a quantitative claim of the vitamin content and all products in the study contain only TA. Vitamin $\mathrm{E}$ acts primarily as a lipophilic radical-scavenging antioxidant and suppresses chain initiation and/or chain propagation steps by donating its 6-phenolic hydrogen to the oxygen radicals. The hydroxyl group of $\mathrm{T}$ can be protected from oxidation by esterification with a carboxyl group of an organic acid such as acetic acid to yield the prodrug TA, which has to liberate the active form (T) by an enzyme-catalyzed hydrolytic process after application to 
Table 2. Mean ( \pm RSD) percent of $T$ or TA permeated on application of commercial cosmetic products (A-D) or experimental formulations $(\mathrm{F} 1, \mathrm{~F} 2)$ to neonatal rats $(\mathrm{n}=3)$

\begin{tabular}{llllr}
\hline Product & $\begin{array}{l}\text { Mean quantity } \\
\text { of cream } \\
\text { applied, mg }\end{array}$ & $\begin{array}{l}\text { Mean quantity of } \\
\text { vitamin in applied } \\
\text { cream, } \mu \mathrm{g}\end{array}$ & $\begin{array}{l}\text { Amount of } \\
\text { permeated } \\
\text { vitamin, } \mu \mathrm{g}\end{array}$ & \% of T/TA \\
\hline Product A (TA only) & 112.60 & 113.12 & 0.00 & 0.00 \\
Product B (TA only) & 93.29 & 531.11 & $66.92 \pm 16.39$ & 12.60 \\
Product C (TA only) & 144.93 & 826.12 & $82.86 \pm 14.10$ & 10.03 \\
Product D (TA only) & 144.7 & 680.1 & $77.53 \pm 16.11$ & 11.40 \\
F1 (T only) & 114.87 & 643.25 & $27.66 \pm 12.72$ & 4.30 \\
F2 (TA only) & 137.57 & 797.89 & $39.42 \pm 5.53$ & 4.94 \\
\hline
\end{tabular}

the skin [14]. The free -OH group in $\mathrm{T}$ is easily oxidized by atmospheric oxygen, and that is why most cosmetic manufacturers prefer TA in formulations rather than $\mathrm{T}$ to guard against loss of content.

The solubility of the drug plays an important role in the in vitro transdermal permeation studies when static diffusion cells are used. With the use of static diffusion cells, the drug solubility is high enough to maintain the sink conditions. However, in the present in vitro transdermal permeation studies, dynamic diffusion cells were used to maintain the required sink conditions. Apparently several investigators [18-20] prefer the use of dynamic diffusion cells to simulate transdermal drug delivery using aqueous phosphate buffer as the receptor fluid.

The failure of T and TA in the tested products to reach the receiver compartment through the stratum corneum, as indicated by the in vitro data in this study, may be due to poor drug release from the different types of products or failure of the vitamin molecules to cross the stratum corneum. Therefore, in vivo experiments in neonatal rats were carried out to highlight and answer many of the questions associated with the in vitro permeation study. It is worth mentioning here that the used method of anal$y$ sis is sensitive as indicated by a limit of detection for $\mathrm{T}$ and TA of 200 and $300 \mathrm{ng} / \mathrm{ml}$, respectively, while the limit of quantitation was 250 and $400 \mathrm{ng} / \mathrm{ml}$, respectively.

The in vivo experiments revealed the absence of T/TA in the epidermis following the repeated application of the different types of products to the dorsal side of neonatal rats. By repeating the same experiment but analyzing for the vitamin in the full-thickness skin, the vitamin was detected in the deeper skin layers. This finding confirmed a previous report [5] pointing out that $\mathrm{T}$ is distributed in the skin with the highest levels in the deeper layers. The commercial preparation (product A) containing the least TA concentration (0.12\%) did not result in any detectable concentration in the rat skin. Therefore, it is recommended to include higher concentrations of T/TA in such formulations to increase the driving force of penetration. Alternatively, the cosmetic formulation can be modified to enhance the highly lipid-soluble $\mathrm{T}$ permeation using novel drug delivery carriers. It is worth mentioning here that although permeation of TA was observed, no free $\mathrm{T}$ could be detected. This finding indicates that the enzymatic activity under the experimental conditions was unable to convert the inactive prodrug TA into the biologically active free $\mathrm{T}$. This finding was also demonstrated previously in a human study [13], in which the acetate ester of tocopherol showed no evidence of conversion to the biologically active form, $\mathrm{T}$, despite adequate absorption into the skin. A study by Rangarajan and Zatz [14] on the metabolic conversion of TA into $\mathrm{T}$ in skin showed that permeation and metabolism of TA was highly dependent on the delivery system, reemphasizing the importance of formulation in cosmetic preparations [14]. The importance of active versus inactive forms, appropriate concentration, consistent delivery and product stability remain hurdles which most of the published literature has yet to cross [12]. Finally, the results of the present study demonstrate the need to suggest/revisit regulations pertaining to such products.

\section{Conclusions}

TA and T exhibited variable skin penetration and TA did not transform into $\mathrm{T}$ under the present experimental conditions. In addition to other factors, the cosmetic/ therapeutic benefit of cosmetics due to $\mathrm{T}$ depends on the concentration used. Therefore, a minimum concentra- 
tion of $1.0 \%$ may be recommended. Marketed products exhibited variable contents of TA. Therefore, the cosmetic/therapeutic efficiency depends on release and permeation as well as the ability of the human body to convert the prodrug TA into the active form T. Further studies to optimize such formulations are required to improve $\mathrm{T}$ stability and hence allow its formulation in place of the prodrug TA in order to achieve the expected cosmetic/ therapeutic outcomes.

\section{Acknowledgments}

This work was supported by Kuwait University Research Grant No. PP01/05. The authors appreciate the technical help of Dr. F. Bandarkar, Mrs. E. Abraham, Mr. S. Abraham, Mrs. D. Nabil as well as Mrs. L. Varghese and Mrs. A. Thomas.

\section{References}

1 Kumano Y, Nishiyama S, Ozawa T, Takahashi M: Skin care products; in Rieger MM (ed): Harry's Cosmeticology, ed 3. New York, Chemical Publishing, 2000, pp 351-392.

$\checkmark 2$ Nachbar F, Korting HC: The role of vitamin $\mathrm{E}$ in normal and damaged skin. J Mol Med 1995;73:7-17.

3 Gehring W, Fluhr J, Gloor M: Influence of vitamin E acetate on stratum corneum hydration. Arzneimittelforschung 1998;48: $772-775$.

4 Gensler HL, Magdaleno M: Topical vitamin E inhibition of immunosuppression tumorigenesis induced by ultraviolet irradiation. Nutr Cancer 1991;15:97-106.

$\checkmark 5$ Shindo Y, Witt E, Han D, Epstein W, Packer L: Enzymic and non-enzymic antioxidants in epidermis and dermis of human skin. J Invest Dermatol 1994;102:122-124.

$\checkmark 6$ Thiele JJ, Schroeter C, Hsieh SN, Podda M, Packer L: The antioxidant network of the stratum corneum. Curr Probl Dermatol 2001;29:26-42.

$>7$ Ricciarelli R, Maroni P, Ozer N, Zingg J-M, Azzi A: Age-dependent increase of collagenase expression can be reduced by alpha-tocopherol via protein kinase C inhibition. Free Radic Biol Med 1999;27:729-737.
8 Zettersten EM, Ghadially D, Feingold D, Crumine A, Elias PM: Optical ratios of topical stratum corneum lipids improve barrier recovery in chronologically aged skin. Acad Dermatol 1997;37:403-408.

$\checkmark 9$ Bisset DL, Chatterjee R, Hannon DP: Photoprotective effect of superoxide-scavenging antioxidants against ultraviolet radiationinduced chronic skin damage in the hairless mouse. Photodermatol Photoimmunol Photomed 1989;7:56-62.

10 Bisset DL, Hillebrand GG, Hannon DP: The hairless mouse as a model of skin photoaging; its use to evaluate photoprotective materials. Photodematol Photoimmunol Photomed 1989;6:228-233.

11 Darr D, Dunston S, Faust H, Pinnell S: Effectiveness of antioxidants (vitamin $\mathrm{C}$ and $\mathrm{E}$ ) with and without sunscreens as topical photoprotectants. Acta Derm Venereol 1996;76: 264-268.

12 Chiu A, Kimball AB: Topical vitamins, minerals and botanical ingredients as modulators of environmental and chronological skin damage. Br J Dermatol 2003;149:681691.

13 Alberts DS, Goldman R, Xu M J, Dorr RT, Quinn J, Welch K, Guillen-Rodriguez J, Aickin M, Peng YM, Loescher L, Gensler H: Disposition and metabolism of topically administered alpha-tocopherol acetate: a common ingredient of commercially available sunscreens and cosmetics. Nutr Cancer 1996;26:193-201.
4 Rangarajan M, Zatz JL: Effect of formulation on the delivery and metabolism of alpha-tocopheryl acetate. J Cosmet Sci 2001;52:225236.

15 Schwartz RA, Centurion SA: Cosmeceuticals. http://emedicine.medscape.com/article/1067778-overview, updated June 2010 (accessed September 2, 2010).

-16 Nada A, Krishnaiah YSR, Zaghloul A, Khattab I: Analysis of vitamin E in cosmetic preparations by HPLC. J Cosmet Sci 2010;61:353365 .

17 Zhao K, Singh J: In vitro percutaneous absorption of propranolol hydrochloride through porcine epidermis by terpenes/ethanol. J Control Release 1999;62:359-366.

18 Patel MR, Patel RB, Parikh JR, Solanki AB, Patel BG: Investigating effect of microemulsion components: in vitro permeation of ketoconazole. Pharm Dev Technol 2010, E-pub ahead of print.

19 Gwak HS, Chun IK: Effect of vehicles and penetration enhancers on the in vitro percutaneous absorption of tenoxicam through hairless mouse skin. Int J Pharm 2002;236: 57-64.

20 Trotta M, Morel S, Gasco MR: Effect of oil phase composition on the skin permeation of felodipine from $\mathrm{o} / \mathrm{w}$ microemulsions. Pharmazie 1997;52:50-53. 